\title{
Analysis of MDR1 Polymorphisms Among HIV- Infected Individuals on ARV Therapy from Western India
}

HariOm Singh ( $\square$ hariomsgpgims@gmail.com )

National AIDS Research Institute

Dharmesh Samani

National AIDS Research Institute

Vijay Chauware

National AIDS Research Institute

T.N Dhole

Sanjay Gandhi Post Graduate Institute of Medical Sciences

Research article

Keywords: ARV-associated hepatotoxicity, Genetic predisposition, NNRTI regimen, Multidrug-resistant-1, HIV Infected Individuals

Posted Date: August 13th, 2020

DOl: https://doi.org/10.21203/rs.3.rs-51053/v1

License: (9) This work is licensed under a Creative Commons Attribution 4.0 International License. Read Full License 


\section{Abstract}

Background: MDR1 is involved in the transport of numerous drugs. Polymorphism of MDR1 is linked with the treatment outcome. ARV regimen is being used to manage the progression of HIV infection. Ethnic disparities have been observed in the distribution of MDR1 genotypes.

Methods: MDR1 polymorphism (1236 C/T, $3435 \mathrm{C} / \mathrm{T}$ ) was genotyped in 34 individuals with ARVassociated hepatotoxicity, $131 \mathrm{HIV}$-infected, and one-fifty-five healthy by utilization of PCR-RFLP.

Results: Haplotype TC exposed the greater risk for hepatotoxicity severity when compared between individuals with hepatotoxicity and HIV infected $(\mathrm{OR}=1.96, \mathrm{P}=0.06)$. While haplotypes TT and CC bared a reduce risk for hepatotoxicity severity $(\mathrm{OR}=0.16, \mathrm{P}=0.006 ; \mathrm{OR}=0.46, \mathrm{P}=0.06)$. Haplotype TT and $\mathrm{CC}$ displayed a decrease risk of hepatotoxicity severity while compared between individuals with hepatotoxicity and healthy $(\mathrm{OR}=0.09, \mathrm{P}=0.003 ; \mathrm{OR}=0.34, \mathrm{P}=0.03)$. A higher occurrence of MDR1 1236TT genotype was seen among patients with hepatotoxicity who consumed alcohol ( $28.6 \%$ versus $14.8 \%$, $\mathrm{OR}=1.50$ ). In patients with hepatotoxicity taking nevirapine, there was an increased incidence of MDR1 1236TT genotype in contrast with efavirenz (21.7\% versus 9.1\%, OR=2.11). In HIV-infected people taking nevirapine, MDR1 1236CT, 1236TT genotypes found to be increased compared with efavirenz (43.7\% versus $33.3 \%$, OR=1.66; $12.6 \%$ versus $8.3 \%, \mathrm{OR}=1.96$ ). A higher occurrence of MDR1 1236TT genotype has happened in hepatotoxicity cases having both alcohol and nevirapine $(40.0 \%$ versus $16.67 \%$, $\mathrm{OR}=2.21$ ).

Conclusion: MDR1 haplotypes may have an influence on the severity of hepatotoxicity. Individual utilizing nevirapine and alcohol with MDR1 1236TT and 3435CT genotypes may have combined effect on vulnerability of severity of hepatotoxicity and progression of HIV infection

\section{Background}

Antiretroviral (ARV) regimen is being extensive utilized for the management of HIV patients, the death rate among individuals with HIV is constantly increasing. Long-term efficacy and toxicity are significant issues of worry with the ARV regimen. Hepatotoxicity is an adverse outcome of ARV drugs in HIV-infected individuals [1,2]. A higher occurrence of hepatotoxicity was seen with the utilization of nevirapine-based regimen than the efavirenz-based regimen [3]. The shown occurrence of hepatotoxicity because of nevirapine was 3.19\% [4]. In another investigation, the stated occurrence of grade 3 or 4 hepatotoxicity had $10.8 \%$ in the patients treated with efavirenz and $8.9 \%$ in patients treated with nevirapine (5). $A B C B 1$ is one of the universal adenosine triphosphate (ATP)-binding cassette (ABC) genes is responsible for cell homeostasis [6-8].

$A B C B 1$ gene is situated on chromosome 7q21. It is a part of the MDR subfamily [8]. ABCB1 protein is expressed in a few tissues including epithelial cells of the blood-brain barrier $[9,10]$ and has appeared to transport numerous drugs [11]. P-glycoprotein (P-GP), a transmembrane transporter protein, is encoded by the MDR1.P-gp system of ATP-subordinate efflux transports outside particles including drugs from 
intracellular to the extracellular matrix [12-14]. The variation in P-gp expression may vary the function, thus affect the transport of nevirapine (NVP) [15]. The absorption and penetration of efavirenz (EFV) and NVP are impacted by the P-gp expression [16]. Chelule et al., (2003) have shown the commonness of MDR1 3435CC genotype had $85.9 \%$ in Africans, $41.70 \%$ in Indians, and $35.7 \%$ in whites of Kwazulu-Natal, South Africa, respectively. In the African population, the predominance of the MDR1 3435CC genotype showed overexpression of P-glycoprotein, while in patients with TT genotype it was demonstrated a lower expression of P-GP $[17,18]$. Studies have been reported a significant increase of CD 4 cell counts with MDR1 3435TT genotype in HIV patients [19, 20]. Haas et al., (2005) proposed that MDR1 3435C/T polymorphism was not linked to efavirenz exposure [19]. Salem et al., (2014) suggested that MDR1 3435C/T polymorphism had no impact on efavirenz clearance [21]. Zhu et al., (2013) proposed that polymorphisms of MDR1 3435T/C and 2677T/G was linked to the response of nevirapine treatment $(\mathrm{P}=$ $0.031, \mathrm{P}=0.001$ ) and could help to predict the drug response in HIV patients [22]. MDR1 3435TT genotype among individuals treated with EFV or nelfinavir was connected with a more elevated level of CD4 + count than the CT/CC genotypes [22]. Leschziner et al., (2007) recommended that MDR1 3435 TT genotype was linked with higher adverse outcome of 3TC and NVP treatment than EFV treatment [23]. Ritchie et al., (2006) indicated a significant reduced risk of hepatotoxicity with NNRTI-containing regimens in the presence of MDR1 3435C/T polymorphism [24]. The link between polymorphism of MDR1 3435C/T and nevirapine induced hepatotoxicity was documented by studies [24, 25].

Few studies have shown a link between MDR1 polymorphism and adverse outcome of ARV drug and other studies do not suggest the link. Moreover, the link between MDR1 polymorphism and ARV-related hepatotoxicity has not been examined from India. Thus, we analyzed the relationship between MDR1 $(1236 \mathrm{C} / \mathrm{T}$ and $3435 \mathrm{C} / \mathrm{T})$ polymorphism and hepatotoxicity induced by ARV regimen.

\subsection{Methods}

\subsection{Subjects}

A case-control design was undertaken between August 2014 to September 2017. The number of individuals with HIV infection experienced a liver function test (LFT) was one hundred sixty-five. Out of which, thirty-four individuals of hepatotoxicity and one thirty-one individual with no hepatotoxicity were affirmed and one hundred fifty-five healthy people age-matched were recruited. The patients were enlisted from the clinic of the National Institute of AIDS Research, Pune. Tuberculosis, Hepatitis B and C, immune reconstitution syndrome, untreated opportunistic infections, and other known hepatotoxic medications have been excluded from the hepatotoxicity case group. At the same time, one hundred fifty-five people (those from a similar family have been excluded) lacking diseases for example, TB, Hepatitis B, C, and HIV, have been recruited as controls. Clinical information was noted through the reviews of case records, questionnaires, and personal interviews. The status of the liver enzymes was assessed by the utilization of LFT. In patients with hepatotoxicity, males with SGOT > 93.8 U/ml, Alkaline phosphatase $>550.8 \mathrm{U} / \mathrm{ml}$, total bilirubin $>3.22 \mathrm{mg} / \mathrm{ml}$, SGPT $>229.5 \mathrm{U} / \mathrm{ml}$ and females with SGOT $>163.2 \mathrm{U} / \mathrm{ml}$, Alkaline phosphatase $>550.8 \mathrm{U} / \mathrm{ml}$, total bilirubin $>3.22 \mathrm{mg} / \mathrm{ml}$, SGPT $>173.4 \mathrm{U} / \mathrm{ml}$ were considered. In HIV- 
infected male and female controls, SGOT $<32 \mathrm{U} / \mathrm{ml}$, Alkaline phosphatase $<108 \mathrm{U} / \mathrm{ml}$, total bilirubin $<1$. $24 \mathrm{mg} / \mathrm{ml}$, and SGPT $<34 \mathrm{U} / \mathrm{ml}$ were considered. FACS analysis was utilized to estimate the CD $4+$ count. The stages of patients were characterized based on current CD4 status. CD4 + cell count ranges from < 200 cells $/ \mathrm{mm} 3,201-350$ cells $/ \mathrm{mm} 3$, and $>350$ cells $/ \mathrm{mm} 3$ were considered as advanced, intermediate, and early stage of HIV infection. HBsAg and hepatitis $C$ testing was completed by ELISA with the Ortho HCV ELISA test system. A questionnaire was utilized to record the usage of tobacco and alcohol. The ethical endorsement was taken from the Ethics Committee, National AIDS Research Institute, Pune, India (Reference number: August 28, 2013, EC/NARI/Genetic Susceptibility/13-14/146) and written consents has been taken from every single qualified subject.

\subsection{Extraction of DNA}

The collection of $2 \mathrm{ml}$ peripheral blood was done from all subjects and put away at $-70^{\circ} \mathrm{C}$ until DNA extraction. The DNA extraction has been completed from blood leukocytes pellets utilizing the QIAamp DNA Mini Kit according to the guidance of the company.

\subsection{Genotyping}

Restriction fragment length polymorphism (RFLP) analysis was utilized to genotype the MDR1 (1236 C/T and $3435 \mathrm{C} / \mathrm{T}$ ) polymorphism. Primers to amplify the MDR1 (C1236T and C3435T) polymorphism were utilized as designated by the previous report [26]. PCR was performed in a volume of $20 \mu$. The PCR conditions for amplification of MDR1 C1236T and C3435T polymorphisms were used as described previously [27]. A thermocycler was utilized to complete all the reactions. PCR products were visualized by ethidium bromide staining. The PCR products of MDR1 C1236T and C3435T polymorphism was digested by utilization of restriction enzymes Haelll and $\mathrm{Mbol}$ at $37^{\circ} \mathrm{C}$ for 16 hours separately. $10 \%$ polyacrylamide gel along with molecular weight markers was utilized to the genotype of MDR1 C1236T and C3435T polymorphism. The sequences and location of SNP were employed for genotyping of MDR1 C1236T and C3535T polymorphisms. Genotypes were: for MDR1 C1236T: 93 and 87 bp for CC, 87, 58, and $35 \mathrm{bp}$ for TT, and 93, 87, 58, and $35 \mathrm{bp}$ for CT, for MDR1 C3435T: 130 and $76 \mathrm{bp}$ for CC, $206 \mathrm{bp}$ for TT and 206, 130, and $76 \mathrm{bp}$ for CT. Additional staff of the laboratory was done re-genotyping in $20 \%$ samples to check the disparities in genotyping. The genotyping mistake was cross-verified by DNA sequencing in $10 \%$ of samples.

\subsection{Statistical examination}

The age variable was communicated as mean \pm standard deviation (SD). Hardy-Weinberg equilibrium was examined by the utilization of Chi-Square Goodness - of - fit test in healthy people. Fisher's exact test was utilized to analyze the genotype frequencies between groups. Logistic regression was utilized to compute the odds ratios (ORs) and 95\% confidence interval (CI). SPSS (SPSS Inc) programming form 17.0 was utilized for statistical examination and the two-sided value was taken as a test of statistical significance. A p-value under $\leq 0.05$ was considered for significance. SNPStats online software was utilized to compare the frequency of haplotypes among groups (25). Linkage disequilibrium (LD) was 
analyzed between both the loci by computing the relative LD value $\left(D^{\prime}\right)$ as $D^{\prime}=$ Dij/ Dmax (28). The Dij value was compared between various groups by the comparison of confidence intervals. 3.0

\subsection{Results}

An aggregate of 165 individuals of HIV infection incorporating thirty-four individuals with hepatotoxicity, one-thirty-one HIV-infected and one-fifty-five healthy were used to analysis the polymorphism of MDR1. The mean age \pm SD of individuals with hepatotoxicity, HIV infected, and healthy have been $37.24 \pm 3.29$, $40.27 \pm 2.45$, and $37.25 \pm 6.30$ years. The demographic profiles of the participants are outlined in Table 1 . 
Table 1

Characteristics of individuals of ARV related hepatotoxicity, HIV infected and healthy controls

\begin{tabular}{|c|c|c|c|}
\hline Subjects & $\begin{array}{l}\text { Individual of ARV- related hepatotoxicity } \\
\text { (Grade III and IV) }\end{array}$ & $\begin{array}{l}\text { Individual of HIV } \\
\text { infection }\end{array}$ & $\begin{array}{l}\text { Healthy } \\
\text { controls }\end{array}$ \\
\hline Number & $N=34$ & $N=131$ & $N=155$ \\
\hline $\begin{array}{l}\text { Mean Age } \\
\text { (Range) }\end{array}$ & $35.14 \pm 8.96$ & $39.29 \pm 1.34$ & $36.75 \pm 8.50$ \\
\hline Females & $16(47.05)$ & $44(33.58)$ & $40(25.80)$ \\
\hline Males & $18(52.94)$ & $84(64.12)$ & 112(72.25) \\
\hline \multicolumn{4}{|c|}{ NNRTI Regimen } \\
\hline $\begin{array}{l}\text { Efavirenz } N= \\
23\end{array}$ & 11 (32.35) & $12(9.16)$ & $\begin{array}{l}\text { Not applicable } \\
\text { (NA) }\end{array}$ \\
\hline Nevirapine & 23(67.64) & $119(90.83)$ & Not applicable \\
\hline \multicolumn{4}{|l|}{$N=142$} \\
\hline \multicolumn{4}{|l|}{ Alcohol habit } \\
\hline User $N=51$ & $7(20.58)$ & $44(33.58)$ & 0 \\
\hline Non user & 27(79.41) & $87(66.41)$ & 0 \\
\hline \multicolumn{4}{|l|}{$N=114$} \\
\hline \multicolumn{4}{|l|}{ Tobacco habit } \\
\hline User $N=50$ & $23(67.64)$ & $27(20.61)$ & 0 \\
\hline $\begin{array}{l}\text { Non user } \mathrm{N}= \\
115\end{array}$ & $11(32.35)$ & 104(79.38) & 0 \\
\hline \multicolumn{4}{|l|}{ CD4 + Status } \\
\hline $0-200(N=95)$ & $16(16.84)$ & 79(83.16) & NA \\
\hline $\begin{array}{l}201-350(\mathrm{~N} \\
=50)\end{array}$ & $17(50)$ & $33(25.19)$ & NA \\
\hline$>350(N=20)$ & $1(2.94)$ & 19(14.50) & NA \\
\hline
\end{tabular}

\subsection{MDR1 polymorphism and ARV-associated hepatotoxicity}


The incidence of polymorphism of MDR 1 in individuals of hepatotoxicity and HIV infected has appeared in Table 2. MDR1 polymorphisms has not been distinct between sufferers with hepatotoxicity and HIV infected. In hepatotoxicity cases, the predominance of MDR1 1236TT genotype was increased $(17.6 \%$ versus $12.2 \%, \mathrm{OR}=1.38,95 \% \mathrm{Cl}: 0.45-4.12, \mathrm{P}=0.57)$. MDR1 3435TT genotype and T allele were underrepresented in hepatotoxicity cases when contrasted with HIV-infected people (35.3\% versus $43.5 \%$, $\mathrm{OR}=0.56,95 \% \mathrm{Cl}: 0.20-1.59, \mathrm{P}=0.28$ and $55.88 \%$ versus $62.59 \%, \mathrm{OR}=0.65, \mathrm{P}=0.13) . M D R 1$ polymorphism were not significantly varied between hepatotoxicity cases and healthy people. 
Table 2

Incidence of MDR1 (1236 C/T and $3435 \mathrm{C} / \mathrm{T})$ genotypes/alleles in individuals of ARV related hepatotoxicity and HIV infected

\begin{tabular}{|c|c|c|c|c|}
\hline $\begin{array}{l}\text { Genotype MDR1 } \\
(1236 \mathrm{C} / \mathrm{T})\end{array}$ & $\begin{array}{l}\text { Individual of ARV- related } \\
\text { hepatotoxicity } \mathrm{N}=34(\%)\end{array}$ & $\begin{array}{l}\text { Individual of HIV } \\
\text { infection } N=131(\%)\end{array}$ & $\begin{array}{l}P \\
\text { Value }\end{array}$ & OR( $95 \% \mathrm{Cl})$ \\
\hline CC & $16(47.1 \%)$ & $59(45.0 \%)$ & & 1(Reference) \\
\hline CT & $12(35.3 \%)$ & $56(42.7 \%)$ & 0.37 & $\begin{array}{l}0.68(0.29- \\
1.60)\end{array}$ \\
\hline TT & $6(17.6 \%)$ & $16(12.2 \%)$ & 0.57 & $\begin{array}{l}1.37(0.45- \\
4.12)\end{array}$ \\
\hline $\mathrm{CT}+\mathrm{TT}$ & $18(52.94)$ & 72(54.96) & 0.63 & $\begin{array}{l}0.82(0.38- \\
1.79)\end{array}$ \\
\hline $\begin{array}{l}\text { MDR1 } \\
(1236 \mathrm{C} / \mathrm{T}) \\
\text { Allele }\end{array}$ & $\begin{array}{l}\text { Individual of ARV- related } \\
\text { hepatotoxicity N = } 68(\%)\end{array}$ & $\begin{array}{l}\text { Individual of HIV } \\
\text { infection N = } 262(\%)\end{array}$ & $\begin{array}{l}\text { P- } \\
\text { Value }\end{array}$ & $\mathrm{OR}(95 \% \mathrm{Cl})$ \\
\hline C & $44(64.71 \%)$ & $174(66.41 \%)$ & & 1(Reference) \\
\hline $\mathrm{T}$ & 24 (35.29\%) & 88 (33.59\%) & 0.91 & $\begin{array}{l}1.03(0.59- \\
1.82)\end{array}$ \\
\hline $\begin{array}{l}\text { Genotype MDR1 } \\
(3435 \mathrm{C} / \mathrm{T})\end{array}$ & $\begin{array}{l}\text { Individual of ARV- related } \\
\text { hepatotoxicity N = } 34(\%)\end{array}$ & $\begin{array}{l}\text { Individual of HIV } \\
\text { infection } \mathrm{N}=131(\%)\end{array}$ & $\begin{array}{l}\text { P- } \\
\text { Value }\end{array}$ & $\mathrm{OR}(95 \% \mathrm{Cl})$ \\
\hline $\mathrm{CC}$ & 8 (23.5\%) & $24(18.3 \%)$ & - & 1(Reference) \\
\hline СT & $14(41.2 \%)$ & $50(38.2 \%)$ & 0.70 & $\begin{array}{l}0.82(0.30- \\
2.25)\end{array}$ \\
\hline TT & $12(35.3 \%)$ & 57 (43.5\%) & 0.28 & $\begin{array}{l}0.56(0.20- \\
1.59)\end{array}$ \\
\hline $\mathrm{CT}+\mathrm{TT}$ & $26(74.47 \%)$ & 107(81.67\%) & 0.42 & $\begin{array}{l}0.68(0.27- \\
1.71)\end{array}$ \\
\hline $\begin{array}{l}\text { MDR1 } \\
\text { (3435 C/T) } \\
\text { Allele }\end{array}$ & $\begin{array}{l}\text { Individual of ARV- related } \\
\text { hepatotoxicity N = } 68(\%)\end{array}$ & $\begin{array}{l}\text { Individual of HIV } \\
\text { infection N = } 262(\%)\end{array}$ & $\begin{array}{l}\text { P- } \\
\text { Value }\end{array}$ & $\mathrm{OR}(95 \% \mathrm{Cl})$ \\
\hline C & $30(44.12 \%)$ & $98(37.40 \%)$ & - & 1(Reference) \\
\hline $\mathrm{T}$ & 38 (55.88\%) & $164(62.59 \%)$ & 0.13 & $\begin{array}{l}0.65(0.37- \\
1.14)\end{array}$ \\
\hline $\begin{array}{l}\text { Genotype MDR1 } \\
(1236 \mathrm{C} / \mathrm{T})\end{array}$ & $\begin{array}{l}\text { Individual of ARV- related } \\
\text { hepatotoxicity N = } 34(\%)\end{array}$ & $\begin{array}{l}\text { Healthy control } \\
\mathrm{N}=155(\%)\end{array}$ & $\begin{array}{l}\mathrm{P} \text { - } \\
\text { Value }\end{array}$ & $\mathrm{OR}(95 \% \mathrm{Cl})$ \\
\hline
\end{tabular}

$\mathrm{N}$, total number of Individual of ARV-related hepatotoxicity (34), HIV infection (131) and healthy controls (155). OR and $95 \% \mathrm{Cls}$ were derived from logistic regression model comparing the homozygous wild-type genotype/allele (CC genotype and C allele for MDR1 $1236 \mathrm{C} / \mathrm{T}$ and $3435 \mathrm{C} / \mathrm{T}$ ) with other genotypes/alleles. 


\begin{tabular}{|c|c|c|c|c|}
\hline $\begin{array}{l}\text { Genotype MDR1 } \\
(1236 \mathrm{C} / \mathrm{T})\end{array}$ & $\begin{array}{l}\text { Individual of ARV- related } \\
\text { hepatotoxicity N = } 34(\%)\end{array}$ & $\begin{array}{l}\text { Individual of HIV } \\
\text { infection } \mathrm{N}=131(\%)\end{array}$ & $\begin{array}{l}P \\
\text { Value }\end{array}$ & $\mathrm{OR}(95 \% \mathrm{Cl})$ \\
\hline $\mathrm{CC}$ & $16(47.1 \%)$ & $69(44.52 \%)$ & & $\begin{array}{l}1 \\
\text { (Reference) }\end{array}$ \\
\hline CT & $12(35.3 \%)$ & 65 (41.94\%) & 0.45 & $\begin{array}{l}0.70(0.27- \\
1.79)\end{array}$ \\
\hline TT & $6(17.6 \%)$ & $21(13.54 \%)$ & 0.98 & $\begin{array}{l}0.99(0.29- \\
3.38)\end{array}$ \\
\hline $\mathrm{CT}+\mathrm{TT}$ & $18(52.94)$ & $86(55.48)$ & 0.55 & $\begin{array}{l}0.77(0.33- \\
1.82)\end{array}$ \\
\hline $\begin{array}{l}\text { MDR1 } \\
(1236 \mathrm{C} / \mathrm{T}) \\
\text { Allele }\end{array}$ & $\begin{array}{l}\text { Individual of ARV-related } \\
\text { hepatotoxicity N = } 68(\%)\end{array}$ & $\begin{array}{l}\text { Healthy Control } \\
N=310\end{array}$ & $\begin{array}{l}\text { P- } \\
\text { Value }\end{array}$ & $\mathrm{OR}(95 \% \mathrm{Cl})$ \\
\hline C & $44(64.71 \%)$ & $203(65.48 \%)$ & - & 1(Reference) \\
\hline $\mathrm{T}$ & 24 (35.29\%) & 107 (34.52\%) & 0.77 & $\begin{array}{l}0.91(0.49- \\
1.71)\end{array}$ \\
\hline $\begin{array}{l}\text { Genotype MDR1 } \\
(3435 \mathrm{C} / \mathrm{T})\end{array}$ & $\begin{array}{l}\text { Individual of ARV- related } \\
\text { hepatotoxicity } \mathrm{N}=34(\%)\end{array}$ & $\begin{array}{l}\text { Healthy Control } \\
\mathrm{N}=155\end{array}$ & $\begin{array}{l}\text { P- } \\
\text { Value }\end{array}$ & OR( $95 \% \mathrm{Cl})$ \\
\hline $\mathrm{CC}$ & 8 (23.5\%) & 34 (21.94\%) & - & 1(Reference) \\
\hline CT & $14(41.2 \%)$ & $67(43.23 \%)$ & 0.24 & $\begin{array}{l}0.52(0.17- \\
1.58)\end{array}$ \\
\hline TT & $12(35.3 \%)$ & $54(34.83 \%)$ & 0.28 & $\begin{array}{l}0.53(0.16- \\
1.69)\end{array}$ \\
\hline $\mathrm{CT}+\mathrm{TT}$ & $26(76.47)$ & $121(78.06)$ & 0.22 & $\begin{array}{l}0.52(0.19- \\
1.46)\end{array}$ \\
\hline $\begin{array}{l}\text { MDR1 } \\
\text { (3435 C/T) } \\
\text { Allele }\end{array}$ & $\begin{array}{l}\text { Individual of ARV- related } \\
\text { hepatotoxicity N = } 68(\%)\end{array}$ & $\begin{array}{l}\text { Healthy Control } \\
N=310\end{array}$ & $\begin{array}{l}\text { P- } \\
\text { Value }\end{array}$ & $\mathrm{OR}(95 \% \mathrm{Cl})$ \\
\hline C & $30(44.12 \%)$ & 135 (43.54\%) & - & 1(Reference) \\
\hline $\mathrm{T}$ & 38 (55.88\%) & 175 (56.45\%) & 0.39 & $\begin{array}{l}0.76(0.41- \\
1.41)\end{array}$ \\
\hline $\begin{array}{l}\mathrm{N} \text {, total number of } \\
\text { controls }(155) \text {. Of } \\
\text { homozygous wild } \\
\text { with other genoty }\end{array}$ & $\begin{array}{l}\text { ndividual of ARV-related he } \\
\text { ind } 95 \% \text { Cls were derived fr } \\
\text { pe genotype/allele (CC ger } \\
\text { s/alleles. }\end{array}$ & $\begin{array}{l}\text { xicity (34), HIV infectio } \\
\text { istic regression model } \\
\text { and } \mathrm{C} \text { allele for MDR } 1\end{array}$ & $\begin{array}{l}\text { 31) and } \\
\text { nparing } \\
6 \mathrm{C} / \mathrm{T} \mathrm{a}\end{array}$ & $\begin{array}{l}\text { healthy } \\
\text { he } \\
\text { d } 3435 \mathrm{C} / \mathrm{T} \text { ) }\end{array}$ \\
\hline
\end{tabular}

\subsection{MDR1 polymorphism in HIV-infected people}

The occurrence of polymorphisms of MDR1 (1236C/T, 3435C/T) in people with HIV infection and healthy is introduced in Table 3. The deviation from Hardy-Weinberg equilibrium in healthy people was 
investigated for MDR1 polymorphism, we found that it was followed $(P=0.36,0.13)$. The distribution of MDR1 polymorphism was alike between HIV-infected and healthy people. HIV-infected people had more occurrence of MDR1 3435TT genotype than healthy people (43.5\% versus $34.83 \%$, OR $=1.24,95 \% \mathrm{Cl}$ : $0.59-2.61, \mathrm{P}=0.57)$. The dispersion of other genotypes and alleles of MDR1 polymorphisms were comparable between both groups. 
Table 3

Incidence of MDR1 (1236 C/T and $3435 \mathrm{C} / \mathrm{T})$ genotypes/alleles in individual of HIV infection and healthy controls.

\begin{tabular}{|c|c|c|c|c|}
\hline $\begin{array}{l}\text { Genotype MDR1 } \\
(1236 \mathrm{C} / \mathrm{T})\end{array}$ & $\begin{array}{l}\text { Individual of HIV infection } \mathrm{N}= \\
131(\%)\end{array}$ & $\begin{array}{l}\text { Healthy } \\
\text { controls } \\
\mathrm{N}=155(\%)\end{array}$ & $\begin{array}{l}P \\
\text { Value }\end{array}$ & $\mathrm{OR}(95 \% \mathrm{Cl})$ \\
\hline CC & $59(45.0 \%)$ & 69 (44.52\%) & - & 1(Reference) \\
\hline CT & $56(42.7 \%)$ & 65 (41.94\%) & 0.65 & $\begin{array}{l}0.87(0.48- \\
1.59)\end{array}$ \\
\hline TT & $16(12.2 \%)$ & 21 (13.54\%) & 0.39 & $\begin{array}{l}0.69(0.29- \\
1.61)\end{array}$ \\
\hline $\mathrm{CT}+\mathrm{TT}$ & 72(54.96) & $86(55.48)$ & 0.49 & $\begin{array}{l}0.82(0.47- \\
1.43)\end{array}$ \\
\hline \multirow[t]{2}{*}{$\begin{array}{l}\text { MDR1 }(1236 \mathrm{C} / \mathrm{T}) \\
\text { Allele }\end{array}$} & $\begin{array}{l}\text { Individual of HIV infection } \mathrm{N}= \\
262(\%)\end{array}$ & $\begin{array}{l}\text { Healthy } \\
\text { controls }\end{array}$ & $\begin{array}{l}\text { P- } \\
\text { Value }\end{array}$ & $\mathrm{OR}(95 \% \mathrm{Cl})$ \\
\hline & & $N=310$ & & \\
\hline C & 174 (66.41\%) & 203 (65.48\%) & - & 1(Reference) \\
\hline $\mathrm{T}$ & 88 (33.59\%) & 107 (34.52\%) & 0.37 & $\begin{array}{l}0.83(0.55- \\
1.25)\end{array}$ \\
\hline \multirow[t]{2}{*}{$\begin{array}{l}\text { Genotype MDR1 } \\
(3435 \mathrm{C} / \mathrm{T})\end{array}$} & $\begin{array}{l}\text { Individual of HIV infection N= } \\
131(\%)\end{array}$ & $\begin{array}{l}\text { Healthy } \\
\text { controls }\end{array}$ & $\begin{array}{l}\text { P- } \\
\text { Value }\end{array}$ & OR( $95 \% \mathrm{Cl})$ \\
\hline & & $N=155$ & & \\
\hline $\mathrm{CC}$ & $24(18.3 \%)$ & 34 (21.94\%) & - & 1(Reference) \\
\hline CT & $50(38.2 \%)$ & 67 (43.23\%) & 0.53 & $\begin{array}{l}0.79(0.37- \\
1.66)\end{array}$ \\
\hline TT & 57 (43.5\%) & 54 (34.83\%) & 0.57 & $\begin{array}{l}1.24(0.59- \\
2.61)\end{array}$ \\
\hline $\mathrm{CT}+\mathrm{TT}$ & 107(81.67) & $121(78.06)$ & 0.97 & $\begin{array}{l}0.99(0.50- \\
1.94)\end{array}$ \\
\hline \multirow[t]{2}{*}{$\begin{array}{l}\text { MDR1 }(3435 \mathrm{C} / \mathrm{T}) \\
\text { Allele }\end{array}$} & $\begin{array}{l}\text { Individual of HIV infection N = } \\
262(\%)\end{array}$ & $\begin{array}{l}\text { Healthy } \\
\text { controls }\end{array}$ & $\begin{array}{l}\text { P- } \\
\text { Value }\end{array}$ & $\mathrm{OR}(95 \% \mathrm{Cl})$ \\
\hline & & $N=310$ & & \\
\hline C & 98 (37.40\%) & 135 (43.54\%) & - & 1(Reference) \\
\hline
\end{tabular}

$\mathrm{N}$, total number of individual of HIV infection (131) and healthy controls (155). OR and $95 \%$ Cls were derived from logistic regression model comparing the homozygous wild-type genotype/allele (CC genotype and C allele for MDR1 $1236 \mathrm{C} / \mathrm{T}$ and $3435 \mathrm{C} / \mathrm{T}$ ) with other genotypes/alleles. 


\begin{tabular}{|c|c|c|c|c|}
\hline $\begin{array}{l}\text { Genotype MDR1 } \\
(1236 \mathrm{C} / \mathrm{T})\end{array}$ & $\begin{array}{l}\text { Individual of HIV infection } N= \\
131(\%)\end{array}$ & $\begin{array}{l}\text { Healthy } \\
\text { controls } \\
\mathrm{N}=155(\%)\end{array}$ & $\begin{array}{l}P \\
\text { Value }\end{array}$ & $\mathrm{OR}(95 \% \mathrm{Cl})$ \\
\hline $\mathrm{T}$ & $164(62.59 \%)$ & 175 (56.45\%) & 0.41 & $\begin{array}{l}1.18(0.80- \\
1.74)\end{array}$ \\
\hline \multicolumn{5}{|c|}{$\begin{array}{l}\mathrm{N} \text {, total number of individual of HIV infection (131) and healthy controls }(155) \text {. OR and } 95 \% \text { Cls were } \\
\text { derived from logistic regression model comparing the homozygous wild-type genotype/allele (CC } \\
\text { genotype and C allele for MDR } 1236 \mathrm{C} / \mathrm{T} \text { and } 3435 \mathrm{C} / \mathrm{T} \text { ) with other genotypes/alleles. }\end{array}$} \\
\hline
\end{tabular}

\subsection{Haplotypes distribution}

We have likewise investigated the occurrence of MDR 1 haplotypes among people of hepatotoxicity, HIVinfected, and healthy people. Haplotype CT $\left(1236^{*} \mathrm{C} / 3435^{\star} \mathrm{T}\right)$ was considered as a reference. Haplotype occurrence has appeared in Table 4 . The incidence of TT haplotype $\left(1236^{*} \mathrm{~T} / 3435^{*} \mathrm{~T}\right)$ had reduced in individuals with hepatotoxicity than HIV-infected $(0.05 \%$ versus $0.22 \%, \mathrm{OR}=0.16,95 \% \mathrm{Cl}: 0.04-0.059, \mathrm{P}=$ $0.0065)$. However, in a similar investigation, the incidence of TC haplotype $\left(1236^{\star} \mathrm{T} / 3435^{\star} \mathrm{C}\right)$ was increased in individuals with hepatotoxicity than HIV-infected $(0.30 \%$ versus $0.11 \%, \mathrm{OR}=1.96,95 \% \mathrm{Cl}$ : $0.98-3.94, \mathrm{P}=0.06)$. The commonness of $\mathrm{CC}(1236 * \mathrm{C} / 3435 * \mathrm{C})$ and TT $(1236 * \mathrm{~T} / 3435 * \mathrm{~T})$ haplotypes was reduced in peoples with hepatotoxicity than healthy $(0.14 \%$ versus $0.30 \%, \mathrm{OR}=0.34,95 \% \mathrm{Cl}: 0.12-$ $0.94, \mathrm{P}=0.039,0.05 \%$ versus $0.22 \%, \mathrm{OR}=0.09,95 \% \mathrm{Cl}: 0.02-0.44, \mathrm{P}=0.0032)$. The incidence of $\mathrm{TC}$ $\left(1236 * \mathrm{~T} / 3435^{*} \mathrm{C}\right)$ haplotype was predominantly higher in patients with hepatotoxicity when contrasted with healthy $(0.30 \%$ versus $0.13 \%, O R=1.94,95 \% \mathrm{Cl}: 0.87-4.37, \mathrm{P}=0.11)$. The incidence of MDR1 haplotypes was not distinguished between individuals of HIV contamination and healthy. 
Table 4

Incidence of MDR1 haplotypes (1236C/T and 3435C/T) in individual of ARV-related hepatotoxicity, HIV infection and healthy controls

\begin{tabular}{|c|c|c|c|c|}
\hline $\begin{array}{l}\text { Haplotype } \\
\text { MDR1 }(1236 \mathrm{C} / \mathrm{T} \text { and } \\
3435 \mathrm{C} / \mathrm{T})\end{array}$ & $\begin{array}{l}\text { Individual of ARV-related } \\
\text { hepatotoxicity }(\mathrm{N}=68)\end{array}$ & $\begin{array}{l}\text { Individual of HIV } \\
\text { infection }(\mathrm{N}=262)\end{array}$ & $\begin{array}{l}P \\
\text { Value }\end{array}$ & $\mathrm{OR}(95 \% \mathrm{Cl})$ \\
\hline CT & 0.51 & 0.41 & - & 1(Reference) \\
\hline CC & 0.14 & 0.26 & 0.062 & $\begin{array}{l}0.46(0.20- \\
1.03)\end{array}$ \\
\hline TT & 0.05 & 0.22 & 0.006 & $\begin{array}{l}0.16(0.04- \\
0.59)\end{array}$ \\
\hline TC & 0.30 & 0.11 & 0.06 & $\begin{array}{l}1.96(0.98- \\
3.94)\end{array}$ \\
\hline $\begin{array}{l}\text { Haplotype } \\
\text { MDR1 }(1236 \mathrm{C} / \mathrm{T} \text { and } \\
3435 \mathrm{C} / \mathrm{T})\end{array}$ & $\begin{array}{l}\text { Individual of ARV-related } \\
\text { hepatotoxicity }(\mathrm{N}=68)\end{array}$ & $\begin{array}{l}\text { Healthy controls } \\
(\mathrm{N}=310)\end{array}$ & $\begin{array}{l}\text { P- } \\
\text { Value }\end{array}$ & $\mathrm{OR}(95 \% \mathrm{Cl})$ \\
\hline CT & 0.51 & 0.35 & - & 1(Reference) \\
\hline $\mathrm{CC}$ & 0.14 & 0.30 & 0.03 & $\begin{array}{l}0.34(0.12- \\
0.94)\end{array}$ \\
\hline TT & 0.05 & 0.22 & 0.003 & $\begin{array}{l}0.09(0.02- \\
0.44)\end{array}$ \\
\hline TC & 0.30 & 0.13 & 0.11 & $\begin{array}{l}1.94(0.87- \\
4.37)\end{array}$ \\
\hline $\begin{array}{l}\text { Haplotype } \\
\text { MDR1 }(1236 \mathrm{C} / \mathrm{T} \text { and } \\
3435 \mathrm{C} / \mathrm{T})\end{array}$ & $\begin{array}{l}\text { Individual of HIV infection } \\
(\mathrm{N}=262)\end{array}$ & $\begin{array}{l}\text { Healthy controls } \\
(\mathrm{N}=310)\end{array}$ & $\begin{array}{l}\text { P- } \\
\text { Value }\end{array}$ & $\mathrm{OR}(95 \% \mathrm{Cl})$ \\
\hline CT & 0.41 & 0.35 & - & 1(Reference) \\
\hline $\mathrm{CC}$ & 0.26 & 0.30 & 0.31 & $\begin{array}{l}0.77(0.46- \\
1.28)\end{array}$ \\
\hline TT & 0.22 & 0.22 & 0.31 & $\begin{array}{l}0.75(0.43- \\
1.31)\end{array}$ \\
\hline TC & 0.11 & 0.13 & 0.43 & $\begin{array}{l}0.77(0.40- \\
1.48)\end{array}$ \\
\hline \multicolumn{5}{|c|}{$\begin{array}{l}\mathrm{N} \text {, total number of allele in HIV- patients with hepatotoxicity }(68) \text {, without hepatotoxicity }(262) \text { and } \\
\text { healthy controls }(310) \text {. OR and } 95 \% \text { Cls were derived from logistic regression model comparing the } \\
\text { haplotype CT with other haplotypes. Significant } P \text { values are shown in bold }(P<0.05)\end{array}$} \\
\hline
\end{tabular}


Table 5

Occurance of MDR1 (1236 C/T and $3435 \mathrm{C} / \mathrm{T}$ ) genotypes in different HIV stages of HIV and healhty controls

\begin{tabular}{|c|c|c|c|c|c|c|c|}
\hline \multirow{2}{*}{$\begin{array}{l}\text { Genotype } \\
\text { MDR1 } \\
(1236 \mathrm{C} / \mathrm{T})\end{array}$} & \multirow{2}{*}{$\begin{array}{l}\text { Healthy } \\
\text { controls } \\
N=155 \\
(\%)\end{array}$} & \multicolumn{2}{|c|}{ Early stage of HIV } & \multicolumn{2}{|c|}{$\begin{array}{l}\text { Intermediate stage of } \\
\text { HIV }\end{array}$} & \multicolumn{2}{|c|}{$\begin{array}{l}\text { Advanced stage of } \\
\text { HIV }\end{array}$} \\
\hline & & $\begin{array}{l}\mathrm{N}=19 \\
(\%)\end{array}$ & OR (P) & $\begin{array}{l}\mathrm{N}=33 \\
(\%)\end{array}$ & OR (P) & $\begin{array}{l}N=79 \\
(\%)\end{array}$ & $\mathrm{OR}(\mathrm{P})$ \\
\hline $\mathrm{CC}$ & $\begin{array}{l}69 \\
(44.52 \%)\end{array}$ & $\begin{array}{l}7 \\
(36.8 \%)\end{array}$ & $\begin{array}{l}1 \\
\text { (Reference) }\end{array}$ & $\begin{array}{l}18 \\
(54.5 \%)\end{array}$ & $\begin{array}{l}1 \\
\text { (Reference) }\end{array}$ & $\begin{array}{l}34 \\
(43.0 \%)\end{array}$ & $\begin{array}{l}1 \\
\text { (Reference) }\end{array}$ \\
\hline CT & $\begin{array}{l}65 \\
(41.94 \%)\end{array}$ & $\begin{array}{l}10 \\
(52.6 \%)\end{array}$ & $1.67(0.36)$ & $\begin{array}{l}8 \\
(24.2 \%)\end{array}$ & $0.43(0.09)$ & $\begin{array}{l}38 \\
(48.1 \%)\end{array}$ & $1.03(0.93)$ \\
\hline TT & $\begin{array}{l}21 \\
(13.54 \%)\end{array}$ & $\begin{array}{l}2 \\
(10.5 \%)\end{array}$ & $0.77(0.77)$ & $\begin{array}{l}7 \\
(21.2 \%)\end{array}$ & $0.93(0.90)$ & $\begin{array}{l}7 \\
(8.9 \%)\end{array}$ & $0.58(0.32)$ \\
\hline \multirow{2}{*}{$\begin{array}{l}\text { Genotype } \\
\text { MDR1 } \\
(3435 \mathrm{C} / \mathrm{T})\end{array}$} & \multirow{2}{*}{$\begin{array}{l}\text { Healthy } \\
\text { controls } \\
N= \\
155(\%)\end{array}$} & \multicolumn{2}{|c|}{ Early stage of HIV } & \multicolumn{2}{|c|}{$\begin{array}{l}\text { Intermediate stage of } \\
\text { HIV }\end{array}$} & \multicolumn{2}{|c|}{$\begin{array}{l}\text { Advanced stage of } \\
\text { HIV }\end{array}$} \\
\hline & & $\begin{array}{l}\mathrm{N}=19 \\
(\%)\end{array}$ & OR (P) & $\begin{array}{l}\mathrm{N}=33 \\
(\%)\end{array}$ & OR (P) & $\begin{array}{l}N=79 \\
(\%)\end{array}$ & OR $(P)$ \\
\hline $\mathrm{CC}$ & $\begin{array}{l}34 \\
(21.94 \%)\end{array}$ & $\begin{array}{l}4 \\
(21.1 \%)\end{array}$ & $\begin{array}{l}1 \\
\text { (Reference) }\end{array}$ & $\begin{array}{l}5 \\
(15.2 \%)\end{array}$ & $\begin{array}{l}1 \\
\text { (Reference) }\end{array}$ & $\begin{array}{l}15 \\
(19.0 \%)\end{array}$ & $\begin{array}{l}1 \\
\text { (Reference) }\end{array}$ \\
\hline CT & $\begin{array}{l}67 \\
(43.23 \%)\end{array}$ & $\begin{array}{l}6 \\
(31.6 \%)\end{array}$ & $0.41(0.23)$ & $\begin{array}{l}12 \\
(36.4 \%)\end{array}$ & $0.80(0.72)$ & $\begin{array}{l}32 \\
(40.5 \%)\end{array}$ & $0.83(0.66)$ \\
\hline TT & $\begin{array}{l}54 \\
(34.83 \%)\end{array}$ & $\begin{array}{l}9 \\
(47.4 \%)\end{array}$ & $0.93(0.92)$ & $\begin{array}{l}16 \\
(48.5 \%)\end{array}$ & $1.56(0.45)$ & $\begin{array}{l}32 \\
(40.5 \%)\end{array}$ & $1.11(0.81)$ \\
\hline
\end{tabular}

\subsection{MDR1 polymorphisms and stages of HIV-1}

The incidence of MDR1 polymorphism between stages of HIV infection and healthy people was examined as outlined in table-5. A reduced frequency of MDR1 1236TT genotype was found in individuals with an intermediate stage of HIV than healthy $(24.2 \%$ versus $41.94 \%, \mathrm{OR}=0.43, \mathrm{P}=0.09)$. The incidence of MDR1 3435CT and 3435TT genotypes did not vary between individuals of stages of HIV infection and healthy.

\subsection{Gene-environment interaction}

The dispersion of MDR1 polymorphism between individuals with hepatotoxicity and HIV infected utilizing tobacco, alcohol, and NNRTI regimen and nonusers appeared in Tables 6-9. The occurrence of polymorphisms of MDR1 (1236C/T and 3435C/T) was not varied between tobacco utilizing people with hepatotoxicity, HIV infected versus nonusers. MDR1 1236TT genotype was overrepresented in tobacco utilizing hepatotoxicity patients than nonusers ( $28.6 \%$ versus $14.8 \%$ ) (Table-6). The incidence of 
MDR11236TT genotype was higher in alcohol devouring people with hepatotoxicity than nonusers (28.6\% versus $14.8 \%, \mathrm{OR}=1.50,95 \% \mathrm{Cl}$ : $0.13-17.35, \mathrm{P}=0.88)$. Moreover, an increased incidence of $3435 \mathrm{CT}$ genotype of MDR1 was observed in HIV-infected people who consumed alcohol than nonusers $(50.0 \%$ versus $32.2 \%, \mathrm{OR}=2.47,95 \% \mathrm{Cl}: 0.79-7.70, \mathrm{P}=0.12)($ Table-7). The genotype $1236 \mathrm{TT}$ of MDR1 was greater in nevirapine taking HIV-contaminated individuals than efavirenz users $(14.1 \%$ versus $8.7 \%$, OR = 1. 93, 95\% Cl: 0. 39- 9.45, $\mathrm{P}=0.42)$. MDR1 1236TT genotype distribution was increased in nevirapine taking patients with hepatotoxicity than efavirenz users $(21.7 \%$ versus $9.1 \%, \mathrm{OR}=2.11,95 \% \mathrm{Cl}$ : $0.18-$ $24.66, \mathrm{P}=0.55)$. The occurrence of MDR1 1236CT 1236TT genotypes was higher in HIV-infected people taking nevirapine than efavirenz users (43.7\% versus $33.3 \%, \mathrm{OR}=1.66,95 \% \mathrm{Cl}: 0.44-6.24, \mathrm{P}=0.45$ and $12.6 \%$ versus $8.3 \%, \mathrm{OR}=1.96,95 \% \mathrm{Cl}: 0.22-17.42, \mathrm{P}=0.55)($ Table-8). In both alcohol and nevirapine taking hepatotoxicity patients, the dispersion of MDR1 1236TT genotype appeared greater when contrasted to alcohol nonusers and nevirapine users $(40.0 \%$ versus $16.67 \%, \mathrm{OR}=2.21,95 \% \mathrm{Cl}$ : $0.17-29.21$, $P=0.55)$. 
Table 6

Incidence of MDR1 (1236 C/T and $3435 \mathrm{C} / \mathrm{T}$ ) genotypes in individuals of ARV related hepatotoxicity and HIV infected utilizing tobacco and non-users

\begin{tabular}{|c|c|c|c|c|}
\hline $\begin{array}{l}\text { Genotype MDR1 } \\
(1236 \mathrm{C} / \mathrm{T})\end{array}$ & $N=7(\%)$ & $\begin{array}{l}\text { Tobacco non- } \\
\text { users } \\
\mathrm{N}=\mathbf{2 7}(\%)\end{array}$ & $\begin{array}{l}P \\
\text { Value }\end{array}$ & $\mathrm{OR}(95 \% \mathrm{Cl})$ \\
\hline \multicolumn{5}{|c|}{ Individuals of ARV related hepatotoxicity } \\
\hline CC & $4(57.1 \%)$ & $12(44.4 \%)$ & - & 1 (Reference) \\
\hline CT & $1(14.3 \%)$ & $11(40.7 \%)$ & 0.30 & $\begin{array}{l}0.28(0.024- \\
3.18)\end{array}$ \\
\hline TT & $2(28.6 \%)$ & $4(14.8 \%)$ & 0.88 & $\begin{array}{l}1.50(0.13- \\
17.35)\end{array}$ \\
\hline $\begin{array}{l}\text { Genotype MDR1 } \\
(3435 \mathrm{C} / \mathrm{T})\end{array}$ & $\begin{array}{l}\text { Tobacco users } \mathrm{N}=7 \\
\text { (\%) }\end{array}$ & $\begin{array}{l}\text { Tobacco non- } \\
\text { users } \\
\mathrm{N}=\mathbf{2 7}(\%)\end{array}$ & $\begin{array}{l}\text { P- } \\
\text { Value }\end{array}$ & $\mathrm{OR}(95 \% \mathrm{Cl})$ \\
\hline $\mathrm{CC}$ & $3(42.9 \%)$ & $5(18.5 .0 \%)$ & - & 1 (Reference) \\
\hline CT & $2(28.6 \%)$ & $12(44.4 \%)$ & 0.37 & $\begin{array}{l}0.37(0.042- \\
3.25)\end{array}$ \\
\hline TT & $2(28.6 \%)$ & $10(37.0 \%)$ & 0.59 & $\begin{array}{l}0.53(0.051- \\
5.40)\end{array}$ \\
\hline \multicolumn{5}{|c|}{ Individuals of HIV infection } \\
\hline $\begin{array}{l}\text { Genotype MDR1 } \\
(1236 \mathrm{C} / \mathrm{T})\end{array}$ & $\begin{array}{l}\text { Tobacco users } \\
\mathrm{N}=\mathbf{4 3}(\%)\end{array}$ & $\begin{array}{l}\text { Tobacco non- } \\
\text { user } \\
\mathrm{N}=\mathbf{8 8}(\%)\end{array}$ & $\begin{array}{l}\mathrm{P}- \\
\text { Value }\end{array}$ & $\mathrm{OR}(95 \% \mathrm{Cl})$ \\
\hline $\mathrm{CC}$ & $19(44.2 \%)$ & $40(45.5 \%)$ & - & 1 (Reference) \\
\hline CT & $19(44.2 \%)$ & $37(42.0 \%)$ & 0.44 & $\begin{array}{l}1.39(0.60- \\
3.20)\end{array}$ \\
\hline TT & $5(11.6 \%)$ & $11(12.5 \%)$ & 0.90 & $\begin{array}{l}1.08(0.31- \\
3.75)\end{array}$ \\
\hline $\begin{array}{l}\text { Genotype MDR1 } \\
(3435 \mathrm{C} / \mathrm{T})\end{array}$ & $\begin{array}{l}\text { Tobacco users } \mathrm{N}=43 \\
\text { (\%) }\end{array}$ & $\begin{array}{l}\text { Tobacco non- } \\
\text { user } \\
\mathrm{N}=\mathbf{8 8}(\%)\end{array}$ & $\begin{array}{l}\mathrm{P}- \\
\text { Value }\end{array}$ & $\mathrm{OR}(95 \% \mathrm{Cl})$ \\
\hline CC & $7(16.3 \%)$ & $17(19.3 \%)$ & - & 1 (Reference) \\
\hline
\end{tabular}

$\mathrm{N}=$ number of subjects, $(\%)=$ frequency of subjects, OR and $95 \%$ Cls were derived from logistic regression model comparing the homozygous wild-type genotype/allele (CC genotype and $\mathrm{C}$ allele for MDR1 $1236 \mathrm{C} / \mathrm{T}$ and $3435 \mathrm{C} / \mathrm{T}$ ) with other genotypes. 


\begin{tabular}{|c|c|c|c|c|}
\hline $\begin{array}{l}\text { Genotype MDR1 } \\
(1236 \mathrm{C} / \mathrm{T})\end{array}$ & $\begin{array}{l}\text { Tobacco users } \\
\mathrm{N}=7 \text { (\%) }\end{array}$ & $\begin{array}{l}\text { Tobacco non- } \\
\text { users } \\
\mathrm{N}=27(\%)\end{array}$ & $\begin{array}{l}P \\
\text { Value }\end{array}$ & $\mathrm{OR}(95 \% \mathrm{Cl})$ \\
\hline CT & 17 (39.5\%) & $33(37.5 \%)$ & 0.74 & $\begin{array}{l}0.80(0.28- \\
2.46)\end{array}$ \\
\hline TT & 19 (44.2\%) & $38(43.2 \%)$ & 0.98 & $\begin{array}{l}1.006(0.43- \\
2.32)\end{array}$ \\
\hline \multicolumn{5}{|c|}{$\begin{array}{l}\mathrm{N}=\text { number of subjects, }(\%)=\text { frequency of subjects, } \mathrm{OR} \text { and } 95 \% \text { Cls were derived from logistic } \\
\text { regression model comparing the homozygous wild-type genotype/allele (CC genotype and } \mathrm{C} \text { allele for } \\
\text { MDR1 } 1236 \mathrm{C} / \mathrm{T} \text { and } 3435 \mathrm{C} / \mathrm{T}) \text { with other genotypes. }\end{array}$} \\
\hline
\end{tabular}


Table 7

Frequency distribution of MDR1 $(1236 \mathrm{C} / \mathrm{T}$ and $3435 \mathrm{C} / \mathrm{T})$ genotypes in hepatotoxicity and HIV pateints with alcohol users and non-users

\begin{tabular}{|c|c|c|c|c|}
\hline $\begin{array}{l}\text { Genotype MDR1 } \\
(1236 \mathrm{C} / \mathrm{T})\end{array}$ & $\begin{array}{l}\text { Alcohol users } \mathrm{N}=7 \\
\text { (\%) }\end{array}$ & $\begin{array}{l}\text { Alcohol non-users N = } \\
27(\%)\end{array}$ & $\begin{array}{l}P \\
\text { Value }\end{array}$ & $\mathrm{OR}(95 \% \mathrm{Cl})$ \\
\hline \multicolumn{5}{|c|}{ Individuals of ARV related hepatotoxicity } \\
\hline $\mathrm{CC}$ & $4(57.1 \%)$ & $12(44.4 \%)$ & - & 1(Reference) \\
\hline CT & $1(14.3 \%)$ & $11(40.7 \%)$ & 0.33 & $\begin{array}{l}0.29(0.025- \\
3.43)\end{array}$ \\
\hline TT & $2(28.6 \%)$ & $4(14.8 \%)$ & 0.88 & $\begin{array}{l}1.50(0.13- \\
17.35)\end{array}$ \\
\hline $\begin{array}{l}\text { Genotype MDR1 } \\
(3435 \mathrm{C} / \mathrm{T})\end{array}$ & $\begin{array}{l}\text { Alcohol users } \mathrm{N}=7 \\
(\%)\end{array}$ & $\begin{array}{l}\text { Alcohol non-users N = } \\
27(\%)\end{array}$ & $\begin{array}{l}\mathrm{P}- \\
\text { Value }\end{array}$ & $\mathrm{OR}(95 \% \mathrm{Cl})$ \\
\hline $\mathrm{CC}$ & $3(42.9)$ & $5(18.5 \%)$ & - & 1(Reference) \\
\hline CT & $3(42.9)$ & $11(40.7 \%)$ & 0.63 & $\begin{array}{l}0.60(0.077- \\
4.73)\end{array}$ \\
\hline TT & $1(14.3)$ & $11(40.7 \%)$ & 0.32 & $\begin{array}{l}0.26(0.018- \\
3.76)\end{array}$ \\
\hline \multicolumn{5}{|c|}{ Individuals of HIV infection } \\
\hline $\begin{array}{l}\text { Genotype MDR1 } \\
(1236 \mathrm{C} / \mathrm{T})\end{array}$ & $\begin{array}{l}\text { Alcohol users } \mathrm{N}= \\
44(\%)\end{array}$ & $\begin{array}{l}\text { Alcohol non-users } \mathrm{N}= \\
87(\%)\end{array}$ & $\begin{array}{l}\mathrm{P}- \\
\text { Value }\end{array}$ & $\mathrm{OR}(95 \% \mathrm{Cl})$ \\
\hline $\mathrm{CC}$ & $23(52.3 \%)$ & $36(41.4 \%)$ & - & 1(Reference) \\
\hline СТ & $18(40.9 \%)$ & $38(43.7 \%)$ & 0.95 & $\begin{array}{l}1.02(0.45- \\
2.35)\end{array}$ \\
\hline TT & $3(6.8 \%)$ & $13(14.9 \%)$ & 0.20 & $\begin{array}{l}0.40(0.098- \\
1.64)\end{array}$ \\
\hline $\begin{array}{l}\text { Genotype MDR1 } \\
(3435 \mathrm{C} / \mathrm{T})\end{array}$ & $\begin{array}{l}\text { Alcohol users } \mathrm{N}= \\
44(\%)\end{array}$ & $\begin{array}{l}\text { Alcohol non-users N = } \\
87(\%)\end{array}$ & $\begin{array}{l}\text { P- } \\
\text { Value }\end{array}$ & $\mathrm{OR}(95 \% \mathrm{Cl})$ \\
\hline $\mathrm{CC}$ & $6(13.6 \%)$ & $18(20.7 \%)$ & - & 1(Reference) \\
\hline CT & $22(50.0 \%)$ & $28(32.2 \%)$ & 0.12 & $\begin{array}{l}2.47(0.79- \\
7.70)\end{array}$ \\
\hline TT & $16(36.4 \%)$ & $41(47.1 \%)$ & 0.81 & $\begin{array}{l}1.15(0.37- \\
3.59)\end{array}$ \\
\hline \multicolumn{5}{|c|}{$\begin{array}{l}\mathrm{N}=\text { number of subjects, }(\%)=\text { frequency of subjects, } \mathrm{OR} \text { and } 95 \% \mathrm{Cls} \text { were derived from logistic } \\
\text { regression model comparing the homozygous wild-type genotype/allele (CC genotype and C allele fo } \\
\text { MDR1 } 1236 \mathrm{C} / \mathrm{T} \text { and } 3435 \mathrm{C} / \mathrm{T}) \text { with other genotypes. }\end{array}$} \\
\hline
\end{tabular}


Table 8

Incidence of MDR1 (1236 C/T and $3435 \mathrm{C} / \mathrm{T})$ genotypes in individuals of ARV-related hepatotoxicity and HIV infected with NNRTIs regimen users and non-users

\begin{tabular}{|c|c|c|c|c|}
\hline $\begin{array}{l}\text { Genotype } \\
\text { MDR1 } \\
(1236 \mathrm{C} / \mathrm{T})\end{array}$ & $\begin{array}{l}\text { Total Individuals of HIV } \\
\text { infection with nevirapine } \\
\text { users } \\
\mathrm{N}=142(\%)\end{array}$ & $\begin{array}{l}\text { Total Individuals of HIV } \\
\text { infection with efavirenz } \\
\text { users } \\
\mathrm{N}=23 \text { (\%) }\end{array}$ & $\begin{array}{l}P \\
\text { Value }\end{array}$ & $\mathrm{OR}(95 \% \mathrm{Cl})$ \\
\hline CC & $63(44.4)$ & $12(52.2)$ & 1 & Reference \\
\hline CT & $59(41.5)$ & $9(39.1)$ & 0.56 & $\begin{array}{l}1.33(0.51- \\
3.47)\end{array}$ \\
\hline TT & $20(14.1)$ & $2(8.7)$ & 0.42 & $\begin{array}{l}1.93(0.39- \\
9.45)\end{array}$ \\
\hline $\begin{array}{l}\text { Genotype } \\
\text { MDR1 } \\
(3435 \mathrm{C} / \mathrm{T})\end{array}$ & $\begin{array}{l}\text { Total Individuals of HIV } \\
\text { infection with with nevirapine } \\
\text { users } \\
\mathrm{N}=142(\%)\end{array}$ & $\begin{array}{l}\text { Total Individuals of HIV } \\
\text { infection with with } \\
\text { efavirenz } \\
\text { users } \\
\mathrm{N}=23(\%)\end{array}$ & $\begin{array}{l}\text { P- } \\
\text { Value }\end{array}$ & $\mathrm{OR}(95 \% \mathrm{Cl})$ \\
\hline $\mathrm{CC}$ & $31(21.8)$ & $1(4.3)$ & 1 & Reference \\
\hline CT & $52(36.6)$ & $12(52.2)$ & 0.063 & $\begin{array}{l}0.14 \\
(0.017- \\
1.11)\end{array}$ \\
\hline TT & $59(41.5)$ & $10(43.5)$ & 0.14 & $\begin{array}{l}0.20 \\
(0.025- \\
1.68)\end{array}$ \\
\hline \multicolumn{5}{|c|}{ Individuals of ARV- related hepatotoxicity } \\
\hline $\begin{array}{l}\text { Genotype } \\
\text { MDR1 } \\
(1236 \mathrm{C} / \mathrm{T})\end{array}$ & $\begin{array}{l}\text { Nevirapine users } \\
N=23(\%)\end{array}$ & $\begin{array}{l}\text { Efavirenz } \\
\text { users } \\
\mathrm{N}=11 \text { (\%) }\end{array}$ & $\begin{array}{l}\mathrm{P}- \\
\text { Value }\end{array}$ & $\mathrm{OR}(95 \% \mathrm{Cl})$ \\
\hline $\mathrm{CC}$ & $11(47.8 \%)$ & $5(45.5 \%)$ & - & 1 (Reference) \\
\hline CT & $7(30.4 \%)$ & $5(45.5 \%)$ & 0.64 & $\begin{array}{l}0.69(0.14- \\
3.35)\end{array}$ \\
\hline TT & $5(21.7 \%)$ & $1(9.1 \%)$ & 0.55 & $\begin{array}{l}2.11(0.18- \\
24.66)\end{array}$ \\
\hline
\end{tabular}

NS, not significant. $\mathrm{N}=$ number of subjects, $(\%)=$ frequency of subjects, OR and $95 \%$ Cls were derived from logistic regression model comparing the homozygous wild-type genotype/allele (CC genotype and $\mathrm{C}$ allele for MDR1 $1236 \mathrm{C} / \mathrm{T}$ and $3435 \mathrm{C} / \mathrm{T}$ ) with other genotypes. 


\begin{tabular}{|c|c|c|c|c|}
\hline $\begin{array}{l}\text { Genotype } \\
\text { MDR1 } \\
(1236 \mathrm{C} / \mathrm{T})\end{array}$ & $\begin{array}{l}\text { Total Individuals of HIV } \\
\text { infection with nevirapine } \\
\text { users } \\
\mathrm{N}=142(\%)\end{array}$ & $\begin{array}{l}\text { Total Individuals of HIV } \\
\text { infection with efavirenz } \\
\text { users } \\
\mathrm{N}=23 \text { (\%) }\end{array}$ & $\begin{array}{l}P \\
\text { Value }\end{array}$ & $\mathrm{OR}(95 \% \mathrm{Cl})$ \\
\hline $\begin{array}{l}\text { Genotype } \\
\text { MDR1 } \\
\text { (3435 C/T) }\end{array}$ & $\begin{array}{l}\text { Nevirapine users } \\
\mathrm{N}=23(\%)\end{array}$ & $\begin{array}{l}\text { Efavirenz } \\
\text { users } \\
\mathrm{N}=11(\%)\end{array}$ & $\begin{array}{l}\text { P- } \\
\text { Value }\end{array}$ & $\mathrm{OR}(95 \% \mathrm{Cl})$ \\
\hline $\mathrm{CC}$ & $7(30.4 \%)$ & $1(9.1 \%)$ & - & 1(Reference) \\
\hline CT & $8(34.8 \%)$ & $6(54.5 \%)$ & 0.18 & $\begin{array}{l}0.19 \\
(0.017- \\
2.11)\end{array}$ \\
\hline TT & $8(34.8 \%)$ & $4(36.4 \%)$ & 0.43 & $\begin{array}{l}0.37 \\
(0.030- \\
4.49)\end{array}$ \\
\hline \multicolumn{5}{|c|}{ Individuals of HIV infection } \\
\hline $\begin{array}{l}\text { Genotype } \\
\text { MDR1 } \\
(1236 \mathrm{C} / \mathrm{T})\end{array}$ & $\begin{array}{l}\text { Nevirapine users } \\
N=119 \text { (\%) }\end{array}$ & $\begin{array}{l}\text { Efavirenz } \\
\text { users } \\
\mathrm{N}=12(\%)\end{array}$ & $\begin{array}{l}\text { P- } \\
\text { Value }\end{array}$ & $\mathrm{OR}(95 \% \mathrm{Cl})$ \\
\hline $\mathrm{CC}$ & $52(43.7 \%)$ & $7(58.3 \%)$ & - & 1(Reference) \\
\hline CT & $52(43.7 \%)$ & $4(33.3 \%)$ & 0.45 & $\begin{array}{l}1.66(0.44- \\
6.24)\end{array}$ \\
\hline TT & $15(12.6 \%)$ & $1(8.3 \%)$ & 0.55 & $\begin{array}{l}1.96(0.22- \\
17.42)\end{array}$ \\
\hline $\begin{array}{l}\text { Genotype } \\
\text { MDR1 } \\
\text { (3435 C/T) }\end{array}$ & $\begin{array}{l}\text { Nevirapine users } \\
N=119 \text { (\%) }\end{array}$ & $\begin{array}{l}\text { Efavirenz } \\
\text { users } \\
\mathrm{N}=12(\%)\end{array}$ & $\begin{array}{l}\text { P- } \\
\text { Value }\end{array}$ & $\mathrm{OR}(95 \% \mathrm{Cl})$ \\
\hline $\mathrm{CC}$ & $24(20.2 \%)$ & $0(0.0 \%)$ & NS & - \\
\hline CT & $44(37.0 \%)$ & $6(50.0 \%)$ & - & 1(Reference) \\
\hline TT & $51(42.9 \%)$ & $6(50.0 \%)$ & 0.81 & $\begin{array}{l}1.16(0.34- \\
3.12)\end{array}$ \\
\hline
\end{tabular}


Table 9

Incidence of MDR1 $(1236 \mathrm{C} / \mathrm{T}$ and $3435 \mathrm{C} / \mathrm{T})$ genotypes in individuals of ARV-related hepatotoxicity and HIV infected with alcohol and NNRTI regimen users and non-users

\begin{tabular}{|c|c|c|c|c|}
\hline $\begin{array}{l}\text { Genotype } \\
\text { MDR1 } \\
(1236 \mathrm{C} / \mathrm{T})\end{array}$ & $\begin{array}{l}\text { Alcohol+ } \\
\text { Nevirapine users } \\
\mathrm{N}=5(\%)\end{array}$ & $\begin{array}{l}\text { Nevirapine users + Alcohol } \\
\text { non-user } \\
\mathrm{N}=18(\%)\end{array}$ & $\begin{array}{l}P \\
\text { Value }\end{array}$ & $\mathrm{OR}(95 \% \mathrm{Cl})$ \\
\hline \multicolumn{5}{|c|}{ Individuals of ARV- related hepatotoxicity } \\
\hline $\mathrm{CC}$ & $3(60.0 \%)$ & $8(44.44 \%)$ & - & 1 (Reference) \\
\hline CT & $0(0.0 \%)$ & 7 (38.89\%) & NS & - \\
\hline TT & $2(40.0 \%)$ & $3(16.67 \%)$ & 0.55 & $\begin{array}{l}2.21(0.17- \\
29.21)\end{array}$ \\
\hline $\begin{array}{l}\text { Genotype } \\
\text { MDR1 } \\
(3435 \mathrm{C} / \mathrm{T})\end{array}$ & $\begin{array}{l}\text { Alcoholt } \\
\text { Nevirapine users } \\
\mathrm{N}=5(\%)\end{array}$ & $\begin{array}{l}\text { Nevirapine users + Alcohol } \\
\text { non-user } \\
\mathrm{N}=18(\%)\end{array}$ & $\begin{array}{l}\text { P- } \\
\text { Value }\end{array}$ & $\mathrm{OR}(95 \% \mathrm{Cl})$ \\
\hline $\mathrm{CC}$ & $3(60.0 \%)$ & $4(22.22 \%)$ & - & 1 (Reference) \\
\hline CT & $1(20.0 \%)$ & 7 (38.39\%) & 0.38 & $\begin{array}{l}0.27(0.015- \\
5.01)\end{array}$ \\
\hline TT & $1(20.0 \%)$ & 7 (38.39\%) & 0.75 & $\begin{array}{l}0.61(0.031- \\
12.05)\end{array}$ \\
\hline $\begin{array}{l}\text { Genotype } \\
\text { MDR1 }\end{array}$ & $\begin{array}{l}\text { Alcohol + Efavirenz } \\
\text { users }\end{array}$ & $\begin{array}{l}\text { Efavirenz users + Alcohol non- } \\
\text { users }\end{array}$ & $\begin{array}{l}\text { P- } \\
\text { Value }\end{array}$ & $\mathrm{OR}(95 \% \mathrm{Cl})$ \\
\hline$(1236 \mathrm{C} / \mathrm{T})$ & $\mathrm{N}=2(\%)$ & $\mathrm{N}=9(\%)$ & & \\
\hline $\mathrm{CC}$ & $1(50.0 \%)$ & $4(44.44 \%)$ & - & 1 (Reference) \\
\hline CT & $1(50.0 \%)$ & $4(44.44 \%)$ & 0.85 & $\begin{array}{l}0.73(0.028- \\
18.97)\end{array}$ \\
\hline TT & $0(0.0 \%)$ & $1(11.12 \%)$ & NS & - \\
\hline $\begin{array}{l}\text { Genotype } \\
\text { MDR1 }\end{array}$ & $\begin{array}{l}\text { Alcohol + Efavirenz } \\
\text { users }\end{array}$ & $\begin{array}{l}\text { Efavirenz users + Alcohol non- } \\
\text { users }\end{array}$ & $\begin{array}{l}\mathrm{P} \text { - } \\
\text { Value }\end{array}$ & $\mathrm{OR}(95 \% \mathrm{Cl})$ \\
\hline (3435 C/T) & $\mathrm{N}=2(\%)$ & $\mathrm{N}=9(\%)$ & & \\
\hline $\mathrm{CC}$ & $0(0.0 \%)$ & $1(11.12 \%)$ & NS & - \\
\hline CT & $2(100 \%)$ & $4(44.44 \%)$ & - & 1(Reference) \\
\hline
\end{tabular}

NS, not significant. $\mathrm{N}$ = number of subjects, $(\%)$ = frequency of subjects, OR and $95 \%$ Cls were derived from logistic regression model comparing the homozygous wild-type genotype/allele (CC genotype and $\mathrm{C}$ allele for MDR1 $1236 \mathrm{C} / \mathrm{T}$ and $3435 \mathrm{C} / \mathrm{T}$ ) with other genotypes. 


\begin{tabular}{|c|c|c|c|c|}
\hline $\begin{array}{l}\text { Genotype } \\
\text { MDR1 } \\
(1236 \mathrm{C} / \mathrm{T})\end{array}$ & $\begin{array}{l}\text { Alcoholt } \\
\text { Nevirapine users } \\
\mathrm{N}=5(\%)\end{array}$ & $\begin{array}{l}\text { Nevirapine users + Alcohol } \\
\text { non-user } \\
\mathrm{N}=18(\%)\end{array}$ & $\begin{array}{l}P \\
\text { Value }\end{array}$ & $\mathrm{OR}(95 \% \mathrm{Cl})$ \\
\hline TT & $0(0.0 \%)$ & $4(44.44 \%)$ & NS & - \\
\hline \multicolumn{5}{|c|}{ individuals of HIV infected } \\
\hline $\begin{array}{l}\text { Genotype } \\
\text { MDR1 }\end{array}$ & $\begin{array}{l}\text { Alcohol + Nevirapine } \\
\text { users }\end{array}$ & $\begin{array}{l}\text { Nevirapine users + Alcohol } \\
\text { non-users }\end{array}$ & $\begin{array}{l}\text { P- } \\
\text { Value }\end{array}$ & $\mathrm{OR}(95 \% \mathrm{Cl})$ \\
\hline (1236 C/T) & $\mathrm{N}=38(\%)$ & $N=81(\%)$ & & \\
\hline CC & $18(47.37 \%)$ & $34(41.98 \%)$ & - & 1 (Reference) \\
\hline CT & $17(44.74 \%)$ & $35(43.20 \%)$ & 0.68 & $\begin{array}{l}1.20(0.50- \\
2.89)\end{array}$ \\
\hline TT & $3(7.89 \%)$ & $12(14.82 \%)$ & 0.40 & $\begin{array}{l}0.54(0.13- \\
2.26)\end{array}$ \\
\hline $\begin{array}{l}\text { Genotype } \\
\text { MDR1 }\end{array}$ & $\begin{array}{l}\text { Alcohol + Nevirapine } \\
\text { users }\end{array}$ & $\begin{array}{l}\text { Nevirapine users + Alcohol } \\
\text { non-users }\end{array}$ & $\begin{array}{l}\mathrm{P}- \\
\text { Value }\end{array}$ & $\mathrm{OR}(95 \% \mathrm{Cl})$ \\
\hline (3435 C/T) & $N=38(\%)$ & $\mathrm{N}=81(\%)$ & & \\
\hline $\mathrm{CC}$ & $6(15.78 \%)$ & $18(22.22 \%)$ & - & 1(Reference) \\
\hline CT & $17(44.74 \%)$ & $27(33.33 \%)$ & 0.23 & $\begin{array}{l}2.04(0.64- \\
6.53)\end{array}$ \\
\hline TT & $15(39.47 \%)$ & $36(44.45 \%)$ & 0.74 & $\begin{array}{l}1.22(0.39- \\
3.84)\end{array}$ \\
\hline $\begin{array}{l}\text { Genotype } \\
\text { MDR1 }\end{array}$ & $\begin{array}{l}\text { Alcohol + Efavirenz } \\
\text { users }\end{array}$ & $\begin{array}{l}\text { Efavirenz users + Alcohol non- } \\
\text { user }\end{array}$ & $\begin{array}{l}\text { P- } \\
\text { Value }\end{array}$ & $\mathrm{OR}(95 \% \mathrm{Cl})$ \\
\hline$(1236 \mathrm{C} / \mathrm{T})$ & $N=6(\%)$ & $N=6(\%)$ & & \\
\hline $\mathrm{CC}$ & $5(83.33 \%)$ & $2(33.33 \%)$ & - & 1(Reference) \\
\hline CT & $1(16.67 \%)$ & $3(50.0 \%)$ & 0.16 & $\begin{array}{l}0.13(0.008- \\
2.18)\end{array}$ \\
\hline TT & $0(0.0 \%)$ & $1(16.67 \%)$ & NS & - \\
\hline $\begin{array}{l}\text { Genotype } \\
\text { MDR1 }\end{array}$ & $\begin{array}{l}\text { Alcohol + Efavirenz } \\
\text { users }\end{array}$ & $\begin{array}{l}\text { Efavirenz users + Alcohol non- } \\
\text { user }\end{array}$ & $\begin{array}{l}\text { P- } \\
\text { Value }\end{array}$ & $\mathrm{OR}(95 \% \mathrm{Cl})$ \\
\hline (3435 C/T) & $N=6(\%)$ & $N=6(\%)$ & & \\
\hline $\mathrm{CC}$ & $0(0.0 \%)$ & 0 & NS & - \\
\hline
\end{tabular}




\begin{tabular}{|lllll|}
\hline $\begin{array}{l}\text { Genotype } \\
\text { MDR1 } \\
(1236 \mathrm{C} / \mathrm{T})\end{array}$ & $\begin{array}{l}\text { Alcohol+ } \\
\text { Nevirapine users } \\
\mathbf{N}=\mathbf{5}(\%)\end{array}$ & $\begin{array}{l}\text { Nevirapine users + Alcohol } \\
\text { non-user } \\
\mathbf{N}=\mathbf{1 8}(\%)\end{array}$ & $\begin{array}{c}\boldsymbol{P} \\
\text { Value }\end{array}$ & OR( 95\%Cl) \\
\hline CT & $5(83.33 \%)$ & $1(16.67 \%)$ & - & 1 (Reference) \\
\hline TT & $1(16.67 \%)$ & $5(83.33 \%)$ & 0.04 & $\begin{array}{l}0.04(0.002- \\
0.83)\end{array}$ \\
\hline $\begin{array}{l}\text { NS, not significant. N = number of subjects, }(\%) \text { frequency of subjects, OR and 95\% Cls were derived } \\
\text { from logistic regression model comparing the homozygous wild-type genotype/allele (CC genotype } \\
\text { and C allele for MDR1 1236 C/T and 3435 C/T) with other genotypes. }\end{array}$ \\
\hline
\end{tabular}

The incidence of 3435CT genotype of MDR1 was greater in nevirapine and alcohol consumed HIVinfected individuals than nevirapine users and alcohol nonusers ( $44.74 \%$ versus $33.33 \%, \mathrm{OR}=2.04$, 95\%Cl: 0. 64-6.53, P = 0. 23) (Table 9).

\subsection{Discussion}

We analyzed the relationship between MDR1 polymorphism and ARV-related hepatotoxicity from Western India. MDR1 encodes for the ATP- dependent membrane efflux transporter (14). The genetic variants that impact patients drugs are substrates of P-glycoproteins. The occurrence of MDR1 polymorphism changes from the population to the population [17]. We analyzed the MDR1 genotypes and haplotypes between individuals infected with HIV and hepatotoxicity.

The occurrence of MDR1 3435C/T polymorphism in our healthy people was identical to the investigations done in European, North India, Turkish, and Asian populations [29-34] and contrasted with the examinations did in Chinese, Iranian and Thailand populations [26, 32, 35]. The genotypic dispersal of MDR1 1236C/T polymorphism in our healthy people was almost alike to the investigation announced from North India [34], however, contrasted with the examinations shown from Indians, Mexicans, Chinese and South Africans populations $[18,30,37,38]$. We have done the genotype-phenotype analysis and found that the MDR11236TT genotype was displayed a hazard for hepatotoxicity severity $(\mathrm{OR}=1.37, \mathrm{P}=$ 0.57). However, due to the small sample size in the hepatotoxicity group, the risk could not reach statistical significance. The low phenotypic expression is dictated by common polymorphisms inside Pgp. People with 3435TT genotype were connected with lower levels of P-gp than CC and CT genotype. MDR1 3435C/T polymorphism was related with the reduced risk of NNRTI-induced liver toxicity [24].

We have examined the relationship of gene-gene interaction to understand the additive impact of MDR1 polymorphism on ARV-related hepatotoxicity. The gene-gene collaboration had a greater effect on gene expression than a single gene [39]. In our investigation, haplotype TC was shown with a greater risk for the severity of hepatotoxicity $(O R=1.96, P=0.06)$. While, haplotypes $T T$ and $C C$ were linked with reduced risk of severity of hepatotoxicity $(O R=0.16, P=0.006 ; O R=0.46, P=0.06 ; O R=0.09, P=0.003 ; O R=0.34$, 
$P=0.03)$. It is likely that individuals with haplotype TC may have prone to the severity of hepatotoxicity, whereas haplotypes TT and CC may have reduced risk for hepatotoxicity severity.

Likewise, we analyzed the relationship between the MDR1 genotype and the stage of HIV infection. In our investigation, the prevalence of MDR1 genotypes did not significantly vary between people of various stages of HIV and healthy. MDR1 1236CT, 1236TT, and 3435CT genotypes were correlated with the HIV disease progression, however, these polymorphisms did not regulate the susceptibility of HIV-1 [40]. Following the treatment of a half year, patients with 3435 TT genotype had raised the CD4 + count [22].

Analysis of the relationship of gene environment was done to take a look at the impact on the etiology of the disease [41, 42]. We had selected a case-only analysis. We did not examine the case-control in light of the fact that in the case-control investigation, cases must be coordinated with the controls in the population [43]. Hence, we utilized the case-only analysis. HIV patient's naïve ART utilizing alcohol has demonstrated a reduction in CD4 + cell count [44]. In women with HIV disease and tobacco utilization, ART response was seen to be diminished [45]. In our examination, the patients of hepatotoxicity utilizing alcohol with MDR11236TT genotype exposed a hazard for severity of hepatotoxicity $(\mathrm{OR}=1.50, \mathrm{P}=0.88)$, while individuals of HIV contamination using alcohol with 3435CT genotype were at higher danger of HIV disease progression ( $\mathrm{OR}=2.47, \mathrm{P}=0.12)$. In hepatotoxic patients utilizing nevirapine and MDR1, 1236TT genotype demonstrated a higher threat for the hepatotoxicity severity $(O R=2.11, P=0.55)$.

Individuals with HIV infection and utilizing nevirapine with MDR1 1236CT, 1236TT genotypes exposed a risk for the progression of HIV infection $(\mathrm{OR}=1.66, \mathrm{P}=0.45 ; \mathrm{OR}=1.96, \mathrm{P}=0.55)$. In people with hepatotoxicity who took both alcohol and nevirapine, MDR1 1236TT genotype exposed the higher vulnerability for hepatotoxicity severity $(\mathrm{OR}=2.21, \mathrm{P}=0.55)$. In people having an infection of HIV with MDR1 3435CT genotype and taking both nevirapine and alcohol showed a vulnerability for the progression of HIV infection ( $\mathrm{OR}=2.04, \mathrm{P}=0.23$ ). This suggests that individuals with MDR1 1236TT and 3435CT genotypes having HIV infection or ARV-related hepatotoxicity have an additive effect on vulnerability of hepatotoxicity severity and progression of HIV disease. Individuals with $3435 \mathrm{~T}$ allele having infection of HIV taking nevirapine were connected with the reduced risk of hepatotoxicity [25]. People with MDR1 1236T and 1235T alleles were related to diminished plasma NNRTI concentration influencing the virological response to HAART [21]. Haas et al., (2005) recommended no significant relationship between $A B C B 1$ variations and plasma EFV concentrations [19].

This work has a few limit points, it can just assess association and not indicate causation. In the beginning, the present investigation was planned for a 1:4 proportion of case controls. However, we were not able to complete to select a similar proportion of controls. Though, we recruited a 1:3 proportion which may be sufficient.

\section{Conclusions}

MDR1 haplotypes may have impact on hepatotoxicity severity. Individual with MDR1 1236TT and 3435CT genotypes in presence of alcohol and nevirapine had an additive effect for vulnerability of 
severity of hepatotoxicity and progression of HIV disease.

MDR1 is associated with drug clearance. MDR1 expression differed in response to NVP and EFV administration. Hence, further examination of the relationship between MDR1 polymorphism and plasma drug concentration would be done with a bigger sample size in different populations. In addition, the correlation of polymorphisms of other drug transporter genes with plasma drug levels is required to comprehend the effect of genetic variants on treatment effect.

\section{Declarations}

\section{Acknowledgment}

We greatly appreciate and acknowledge the clinic In-charges and other supporting staff of NARI for the recruitment of study participants. I would like to thank Asha Krishnaraj for helping in editing the manuscript.

Availability of data and material: On request by email to Corresponding Author

\section{Abbreviation:}

HIV, Human immunodeficiency virus; MDR-1; Multidrug-resistant-1, ABCB1, ATP binding cassette subfamily B member 1; NNRTIs, Non-nucleoside reverse transcriptase inhibitors; ARV, Antiretroviral; ART, Antiretroviral therapy; NVP, Nevirapine; EFV, Efavirenz; SNP, Single nucleotide polymorphism; PCR-RFLP, Polymerase chain reaction- Restriction fragment length polymorphisms; DILI, drug-induced liver injury; ADR, Adverse drug reaction; LFT, Liver function test; SGOT, Serum glutamic oxaloacetic transaminase; SGPT, Serum glutamic pyruvic transaminase; ELISA, Enzyme-linked immunosorbent assay; ABC, ATPbinding cassette

Consent for publication: Yes

Source of funding: study was not supported by any extramural funds.

Competing interests: None

Conflict of interests: Nil

Ethical approval: NARI/EC/ICF version 1.0, dated 28 August 2013

Consent to Participate: Taken

\section{Author's contribution}

HS: Overall supervision

DS: Experimental work 
VC: Manuscript writing

TN D: Clinical input and Critical Review of manuscript

\section{All authors read and approved the final manuscript: Yes}

\section{References}

1. O'Brien ME, Clark RA, Besch CL, Myers L, Kissinger P. (2003). Patterns and correlates of discontinuation of the initial HAART regimen in an urban outpatient cohort. J Acquir Immune.

2. Defic Syndr, 34:407 - 14.

3. Van Dyke RB, Wang L, Williams PL, for the Pediatric ACTGCT. Toxicities Associated with Dual Nucleoside Reverse-Transcriptase Inhibitor Regimens in HIV-Infected Children. The Journal of Infectious Diseases. 2008;198:1599-608.

4. Minzi OM, Irunde $\mathrm{H}$, Moshiro C. HIV patients presenting common adverse drug events caused by highly active antiretroviral therapy in Tanzania. Tanzan J Health Res. 2009;11:5-10.

5. Nagpal M, Tayal V, Kumar S, Gupta U. (2010). Adverse drug reactions to antiretroviral therapy in AIDS patients at a tertiary care hospital in India: A prospective observational study. Indian.

6. J Med Sci. 64:245-52.

7. Mascolini M. (2001). HIV news from Buenos Aires: Part 1-the Zahir and the band-aid. 1st IAS conference on HIV pathogenesis and treatment; July 8-11; Buenos Aires: APAC Mon, $274-89$.

8. Croop JM. P-glycoprotein structure and evolutionary homologies. Cytotechnology. 1993;12:1-32.

9. Jones PM, George AM. The ABC transporter structure and mechanism: perspectives on recent research. Cell Mol Life Sci. 2004;61:682-99.

10. Rosenberg MF, Callaghan R, Ford RC, Higgins CF. Structure of the multidrug resistance P-glycoprotein to $2.5 \mathrm{~nm}$ resolution determined by electron microscopy and image analysis. J Biol Chem. 1997;272:10685-94.

11. Cordon-Cardo C, O'Brien JP, Casals D, Rittman-Grauer L, Biedler JL, Melamed MR, Bertino JR. Multidrug-resistance gene (P-glycoprotein) is expressed by endothelial cells at blood-brain barrier sites. Proc Natl Acad Sci U S A. 1989;86:695-8.

12. Pileri SA, Sabattini E, Falini B, Tazzari PL, Gherlinzoni F, Michieli MG, Damiani D, Zucchini L, Gobbi M, Tsuruo T, et al. Immunohistochemical detection of the multidrug transport protein P170 in human normal tissues and malignant lymphomas. Histopathology. 1991;19:131-40.

13. Sakaeda T, Nakamura T, Okumura K. Pharmacogenetics of MDR1 and its impact on the pharmacokinetics and pharmacodynamics of drugs. Pharmacogenomics. 2003;4:397-410.

14. Ambudkar SV, Dey S, Hrycyna CA, Ramachandra M, Pastan I, Gottesman MM. Biochemical, cellular, and pharmacological aspects of the multidrug transporter. Annu Rev Pharmacol Toxicol. 1999;39:361-98. 
15. Sugawara I, Kataoka I, Morishita Y, Hamada H, Tsuruo T, Itoyama S, Mori S. Tissue distribution of Pglycoprotein encoded by a multidrug-resistant gene as revealed by a monoclonal antibody, MRK 16 . Cancer Res. 1988;48:1926-9.

16. Thiebaut F, Tsuruo T, Hamada H, Gottesman MM, Pastan I, Willingham MC. (1987) Cellular localization of the multidrug-resistance gene product P-glycoprotein in normal human tissues.

17. Proc. Natl Acad Sci U S A 84:7735-8.

18. Stormer E, von Moltke LL, Perloff MD, Greenblatt DJ.(2002). Differential modulation of Pglycoprotein expression and activity by non-nucleoside HIV-1 reverse transcriptase inhibitors.

19. in cell culture. Pharm Res, 19:1038-45.

20. Tozzi V. Pharmacogenetics of antiretrovirals. Antiviral Res. 2010;85(1):190-200.

21. Chelule PK, Gordon M, Palanee T, Page T, Mosam A, Coovadia HM, Cassol S. MDR1 and CYP3A4 polymorphisms among African, Indian, and white populations in KwaZulu-Natal, South Africa. Clin Pharmacol Ther. 2003;74:195-6.

22. Dong Q, Xu B, Tan Y, Liu Z, Tian L, Zhang B, Lin CK, Kung HF, Sung JJ, He ML. The genetic variability of MDR1 C3435T polymorphisms in four Southern Chinese populations. Biomed Pharmacother. 2009;63:658-62.

23. Haas DW, Smeaton LM, Shafer RW, Robbins GK, Morse GD, Labbe L, Wilkinson GR, Clifford DB, D'Aquila RT, De Gruttola V, Pollard RB, Merigan TC, Hirsch MS, George AL Jr, Donahue JP, Kim RB. Pharmacogenetics of long-term responses to antiretroviral regimens containing Efavirenz and/or Nelfinavir: an Adult Aids Clinical Trials Group Study. J Infect Dis. 2005;192:1931-42.

24. Li YH, Wang YH, Li Y, Yang L. MDR1 gene polymorphisms and clinical relevance. Acta Genetica Sinica. 2006;33:93-104.

25. Salem AH, Fletcher CV, Brundage RC. (2014). Pharmacometric characterization of efavirenz developmental pharmacokinetics and pharmacogenetics in HIV-infected children. Antimicrob.

26. Agents Chemother, 58:136 - 43.

27. Fellay J, Marzolini C, Meaden ER, Back DJ, Buclin T, Chave JP, Decosterd LA, Furrer H, Opravil M, Pantaleo G, Retelska D, Ruiz L, Schinkel AH, Vernazza P, Eap CB, Telenti A;Swiss HIV Cohort Study. (2002). Response to antiretroviral treatment in HIV-1-infected individuals with allelic variants of the multidrug resistance transporter 1: a pharmacogenetics study. Lancet, 359:30-6.

28. Leschziner GD, Andrew T, Pirmohamed M, Johnson MR. (2007). ABCB1 genotype and PGP expression, function and therapeutic drug response: a critical review and recommendations for.

29. future research. Pharmacogenomics J.7:154 - 79.

30. Ritchie MD, Haas DW, Motsinger AA, Donahue JP, Erdem H, Raffanti S, Rebeiro P, George AL, Kim RB, Haines JL, Sterling TR. Drug transporter and metabolizing enzyme gene variants and nonnucleoside reverse-transcriptase inhibitor hepatotoxicity. Clin Infect Dis. 2006;43:779-82.

31. Haas DW, Bartlett JA, Andersen JW, Sanne I, Wilkinson GR, Hinkle J, Rousseau F, Ingram CD, Shaw A, Lederman MM, Kim RB, Adult AIDS, Clinical Trials Group. Pharmacogenetics of nevirapine- 
associated hepatotoxicity: an Adult AIDS Clinical Trials Group collaboration. Clin Infect Dis. 2006;43:783-6.

32. Pongstaporn W, Pakakasama S, Chaksangchaichote P, Pongtheerat T, Hongeng S, Permitr S. MDR1 C3435T and C1236T polymorphisms: association with high-risk childhood acute lymphoblastic leukemia. Asian Pac J Cancer Prev. 2015;16:2839-43.

33. Sole X, Guino E, Valls J, Iniesta R, Moreno V. SNPStats: a web tool for the analysis of association studies. Bioinformatics. 2006;22:1928-9.

34. Cox A, Camp NJ, Nicklin MJ, di Giovine FS, Duff GW. An analysis of linkage disequilibrium in the interleukin-1 gene cluster, using a novel grouping method for multiallelic markers. Am J Hum Genet. 1998;62:1180-8.

35. Ghodke Y, Chopra A, Shintre P, Puranik A, Joshi K, Patwardhan B. Profiling single nucleotide polymorphisms (SNPs) across intracellular folate metabolic pathway in healthy Indians. Indian $\mathrm{J}$ Med Res. 2011;133:274-9.

36. Lakhan R, Misra UK, Kalita J, Pradhan S, Gogtay NJ, Singh MK, Mittal B. No association of ABCB1 polymorphisms with drug-refractory epilepsy in a north Indian population. Epilepsy Behav. 2009;14:78-82.

37. Rubis B, Holysz H, Barczak W, Gryczka R, Lacinski M, Jagielski P, et al. Study of ABCB1 polymorphism frequency in breast cancer patients from Poland. Pharmacol Rep. 2012;64(6):1560-6.

38. Shi NJ, Zhang WX, Zhang N, Zhong LN, Wang LP. Correlation of MDR1 gene polymorphisms with anesthetic effect of sevoflurane-remifentanil following pediatric tonsillectomy. Medicine. 2017;96:e7002.

39. Tan EK, Chan DK, Ng PW, Woo J, Teo YY, Tang K, et al. Effect of MDR1 haplotype on risk of Parkinson disease. Arch Neurol. 2005;62(3):460-4.

40. Rustemoglu A, Gumus-Akay G, Yigit S, Tasliyurt T. Analysis of common MDR1 (ABCB1) gene C1236T and C3435T polymorphisms in Turkish patients with familial Mediterranean fever. Genet Mol Res. 2011;10:3411-20.

41. Saidijam M, Mahjub H, Shabab N, Yadegarazari R. (2015).Simultaneous analysis of multidrug resistance 1(MDR1) C3435T, G2677T/A, and C1236T genotypes in Hamadan City population.

42. West of Iran. Iran Biomed J, 19:57-62.

43. Jafar T, Prasad N, Agarwal V, Mahdi A, Gupta A, Sharma RK, et al. (2011). MDR-1 gene polymorphisms in steroid-responsive versus steroid-resistant nephrotic syndrome in children.

44. Nephrol. Dial Transplant, 26:3968-74.

45. Gutierrez-Rubio SA, Quintero-Ramos A, Duran-Cardenas A, Franco-Topete RA, Castro-Cervantes JM, Oceguera-Villanueva A, et al. $1236 \mathrm{C} / \mathrm{T}$ and $3435 \mathrm{C} / \mathrm{T}$ polymorphisms of the ABCB1 gene in Mexican breast cancer patients. Genet Mol Res. 2015;14:1250-9.

46. Masebe TM, Bessong PO, Nwobegahay J, Ndip RN, Meyer D. Prevalence of MDR1 C3435T and CYP2B6 G516T polymorphisms among HIV-1 infected South African patients. Dis Markers. 
2012;32:43-50.

47. Palmer LJ, Cardon LR. Shaking the tree: mapping complex disease genes with linkage disequilibrium. Lancet. 2005;366:1223-34.

48. Bellusci CP, Rocco CA, Aulicino PC, Mecikovsky D, Bologna R, Sen L, et al. MDR1 3435T and 1236T alleles delay disease progression to pediatric AIDS but have no effect on HIV-1 vertical transmission. AIDS. 2010;24:833-40.

49. Deng Y, Newman B, Dunne MP, Silburn PA, Mellick GD. Case-only study of interactions between genetic polymorphisms of GSTM1, P1, T1 and Z1 and smoking in Parkinson's disease. Neurosci Lett. 2004;366:326-31.

50. Hunter DJ, Hankinson SE, Hough H, Gertig DM, Garcia-Closas M, Spiegelman D, et al. (1997). A prospective study of NAT2 acetylation genotype, cigarette smoking, and risk of breast.

51. cancer. Carcinogenesis, 18:2127-32.

52. Greenland S. The effect of misclassification in the presence of covariates. Am J Epidemiol. 1980;112:564-9.

53. Samet JH, Cheng DM, Libman H, Nunes DP, Alperen JK, Saitz R. Alcohol consumption and HIV disease progression. J Acquir Immune Defic Syndr. 2007;46:194-9.

54. Feldman JG, Minkoff H, Schneider MF, Gange SJ, Cohen M, Watts DH, et al. Association of cigarette smoking with HIV prognosis among women in the HAART era: a report from the women's interagency HIV study. Am J Public Health. 2006;96(6):1060-5. 